\title{
Experimental Characterization of the RFID STENTag for Passive Vascular Monitoring
}

\author{
Cecilia Occhiuzzi \\ DISP, University of Roma Tor Vergata, \\ Via del Politecnico, 1 \\ 00133, Roma (ITALY) \\ Email: occhiuzzi@disp.uniroma2.it
}

\author{
Gaetano Marrocco \\ DISP, University of Roma Tor Vergata, \\ Via del Politecnico, 1 \\ 00133, Roma (ITALY) \\ Email: marrocco@disp.uniroma2.it
}

\begin{abstract}
The possibility to monitor biological process in evolution and in particular the state of implanted devices such as vascular stent, has been recently proposed as one of the most challenging and fascinating application of the RFID passive technology. However, several issues are still in question and need to be analyzed in order to evaluate the real medical and sensing effectiveness of the proposed implantable radio sensor. The aim of this work is to experimentally evaluate the robustness of the RFID platform, its accuracy and its reproducibility as well as the effectiveness of the proposed sensing approach in term of human variability and pathology evolution.
\end{abstract}

\section{INTRODUCTION}

The possibility to monitor biological process in evolution and in particular the state of implanted devices such as vascular stent, has been recently proposed as one of the most challenging and fascinating application of the RFID passive technology. Theoretical and experimental investigations demonstrated the feasibility of the idea by means of a first prototype, named STENTag [1]. However, several issues are still in question and need to be analyzed in order to evaluate the real medical and sensing effectiveness of the proposed implantable radio sensor.

The first topic to consider is the robustness of the RFID sensor in terms of measurement accuracy and reproducibility. The sensing metrics in [1] are based on data directly available from commercial readers and could thus suffer from poor resolution and uncertainty, potentially able to invalidate their sensing meaning. Furthermore, being the STENTag designed to be used in non-stationary scenarios, i.e. to be interrogated by means of mobile and personal devices, the effects of the uncertainties in the measurement set-up must be also taken into account.

The second challenging issue is the human variability, e.g the range of possible values characterizing the body shape and size as well as the evolution of the biological process. The STENTag must be able to properly sense the in-stent restenosis (ISR) for what concerns its patterns and timescale and with equal effectiveness and accuracy regardless the patient who hosts it.

Here, all these critical topics are experimentally addressed by means of an articulated measurement campaign over two realistic phantoms of the neck suitable to dynamically reproduce a localized ISR in the carotid and the typical human variability. The repeatability and the accuracy of the measurement are investigated by the help of statistic operators. A multi-frequency analysis is finally adopted to improve the measurement reliability and to further corroborate the reasonableness of the idea.

\section{Measurement CAmpaign}

An optimized prototype of the STENTag has been designed and fabricated according to the guidelines in [1], and experimented in vitro by means of equivalent liquid phantoms (Fig.1).

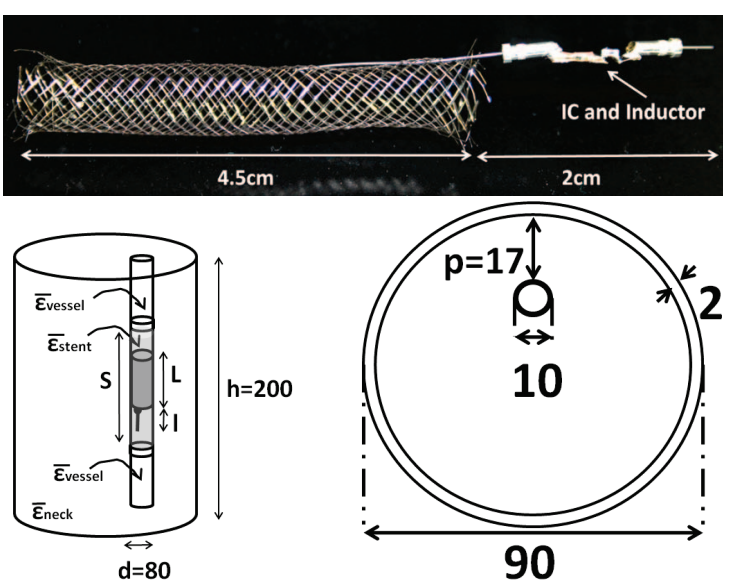

Figure 1. Top) STENTag prototype with a details of the integration of RFID IC and of the inductor. Bottom) Cylindrical model of the neck, the carotid and the ISR (side view and top view). The STENTag is inserted in the middle of the smaller. The ISR is modeled has a localized (shadowed) volume with variable permittivity extending beyond the margins of the stent. Size in [mm].

The neck phantom (Fig.1) is a cylinder of diameter $D=9 \mathrm{~cm}$ and height $h=20 \mathrm{~cm}$ filled by a homogeneous dielectric having the weighted average permittivity of all the main tissues composing the neck, $\left(\bar{\epsilon}_{n e c k}=41.4-j 14.8\right)$, while a smaller offcentered $1 \mathrm{~cm}$-diameter cylinder emulates the human carotid and it is filled by blood-like dielectric $\left(\bar{\epsilon}_{\text {vessel }}\right)$. The STENTag is inserted in the middle of the small cylinder, tightly fitting its internal surface, within a localized volume simulating the diffuse proliferative ISR (one of most severe forms of 
Table I

Re-STENosis: Properties at 870MHz of the Vessel's DieleCtriC

\begin{tabular}{|c|c|c|}
\hline Vessel's filler & $\begin{array}{l}\text { Theoretical Complex } \\
\text { Permittivity }\end{array}$ & $\begin{array}{l}\text { Measured Complex } \\
\text { Permittivity }\end{array}$ \\
\hline $\begin{array}{l}1 \text { - Healthy } \\
\text { vessel } \\
I S R=0 \%\end{array}$ & $\bar{\epsilon}_{1}=61.45-j 31.5$ & $\bar{\epsilon}_{1 m}=57.8-j 33.0$ \\
\hline $\begin{array}{l}\text { 2-Neointimal } \\
\text { proliferation } \\
I S R=50 \%\end{array}$ & $\bar{\epsilon}_{2}=58.22-j 25.3$ & $\bar{\epsilon}_{2 m}=56.17-j 27.6$ \\
\hline $\begin{array}{l}\text { 3-Neointimal } \\
\text { proliferation } \\
I S R=100 \% \\
\text { 4-Plaque }\end{array}$ & $\bar{\epsilon}_{3}=55-j 19.2$ & $\bar{\epsilon}_{3 m}=51.65-j 22.7$ \\
\hline $\begin{array}{l}\text { proliferation } \\
\text { grade } 1\end{array}$ & $\bar{\epsilon}_{4}=48.2-j 17.0$ & $\bar{\epsilon}_{4 m}=46.7-j 20.19$ \\
\hline $\begin{array}{l}5 \text {-Plaque } \\
\text { proliferation } \\
\text { grade } 2\end{array}$ & $\bar{\epsilon}_{5}=41.4-j 14.8$ & $\bar{\epsilon}_{5 m}=41.8-j 17.6$ \\
\hline $\begin{array}{l}\text { 6-Plaque } \\
\text { proliferation } \\
\text { grade } 3\end{array}$ & $\bar{\epsilon}_{6}=27.5-j 10.95$ & $\bar{\epsilon}_{6 m}=27.5-j 10.95$ \\
\hline $\begin{array}{l}\text { 7-Plaque } \\
\text { proliferation } \\
I S R=100 \%\end{array}$ & $\bar{\epsilon}_{7}=14.13-j 12.15$ & $\bar{\epsilon}_{7 m}=14.13-j 12.15$ \\
\hline
\end{tabular}

restenosis characterized by wide lesions ( $>10 \mathrm{~mm}$ long) extending beyond the margins of the stent [2]. Such a district, of height $S=8 \mathrm{~cm}$, is homogeneously filled with a fluid of variable permittivity $\left(\bar{\epsilon}_{\text {stent }}\right)$ suitable to reproduce the various degrees of the ISR (Tab.I), and filled/emptied by means of a proper hydraulic circuit.

The equivalent dielectrics have been produced according to the recipes in [4], e.g mixtures of water, sugar and salt. The measured permittivity of the liquid phantoms (achieved by means of a open-ended coaxial dielectric probe), averaged over 6 samples, are indicated in Tab.I and compared with the expected values from the recipes. All the measurements have been performed within the UHF band (840-960 MHz) by means of a UHF Thing-Magic M5e reader, connected to a $5 \mathrm{~dB}$ gain linear polarized patch antenna (Fig. 2) which is placed at $20 \mathrm{~cm}$ from the neck phantom, as in the simulations. The reader permits to control the input power by $0.5 d B$ steps and the receiving module performs a 8-bit digital conversion of the signals backscattered by the tag.

Fig. 3 shows the curves of the measured turn-on power in the UHF band, versus the change of permittivity of the liquid filling the STENTag. The curves have similar behavior but are clearly distinguishable. The power required to turn on the chip reduces as the stenosis's grade gets worse. A nearly $3 \mathrm{~dB}$ dynamic range is observed at each frequency when moving from the healthy condition to the complete plaque occlusion of the duct.

A more quantitative representation including the error analysis and the comparison with the computer simulations at $870 \mathrm{MHz}$ is given in Fig.4 for what concerns the turn-on power $P_{i n}^{t o}(\Psi)$. The mean value and the standard deviation (the gray region) have been calculated starting from nine measurements at different angular offset (within $\pm 30^{\circ}$ ) between the reader's antenna and the tag, so that real-life uncertainty of

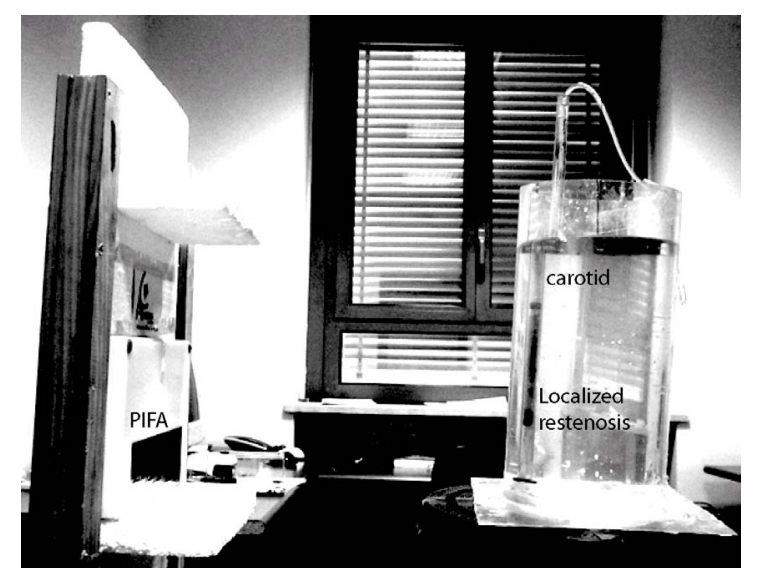

Figure 2. Experimental set-up comprising the liquid phantom resembling the neck where the STENTag has been implanted, and the interrogation $5 \mathrm{~dB}$ patch antenna connected to the Thing-Magic M5e reader (not shown in the figure). The darker area in the central part of the phantom corresponds to the ISR. Such a volume is filled/emptied by means of a proper hydraulic circuit.

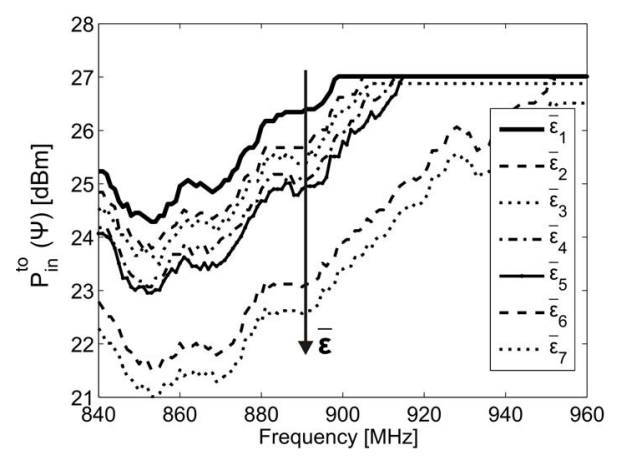

Figure 3. Measured turn-on power in the UHF (World- Wide) Band for the STENTag prototype, versus the change of the liquid permittivity filling the carotid-like pipe.

the measurement set-up are properly reproduced.

The sensitivity to the tag's status is remarkable even in the early modification of the liquid, corresponding to an $I S R=50 \%$ (from $\bar{\epsilon}_{1}$ to $\bar{\epsilon}_{2}$ ) with a monotonic behavior in the entire considered permittivity range. The turn-on measurement is almost invariant with the observation angle with less than $0.5 \mathrm{~dB}$ uncertainty, mainly related to the poor resolution of the reader.

In order to analyze the sensibility of the STENTag to the human variability, a second measurement campaign has been devoted to a thicker neck phantom, resembling a stout subject. The results (Fig. 4) are similar to those of the previous case except for a $3 \mathrm{~dB}$ scaling since the increased size of the phantom and the deeper position of the radio-sensor produce a rise of the losses and consequently of the power required to establish the communication.

The uncertainty in the measured data may be reduced by using all the frequency-domain data available from the 


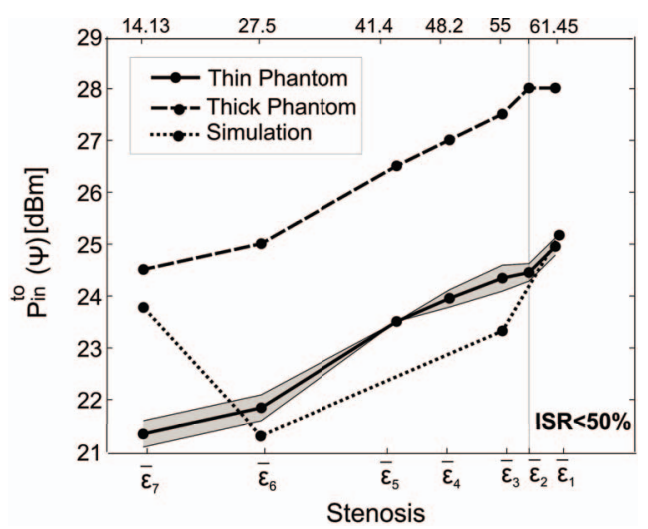

Figure 4. Measured (mean and shadowed uncertainty region) and simulated turn on power for the STENTag prototype at $870 \mathrm{MHz}$ The measured data are sorted according to the real part of the considered complex permittivity.

measurement and introducing an averaging operator over frequency, e.g the normalized scale factor:

$$
\gamma_{\xi}=\frac{<P_{i n}^{t o}(\Psi)-P_{i n}^{t o}\left(\Psi_{0}\right)>}{<P_{i n}^{t o}\left(\Psi_{0}\right)>}
$$

where the " $<>$ " brackets indicate the average over frequency samples and $\Psi_{0}$ the initial stage of the process. Fig.5 shows the averaged $\gamma_{p_{S}}$ for the two phantoms. Since $\gamma$ is a relative parameter, the profiles of the two phantoms are almost overlapped, demonstrating the repeatability of the sensing measurement regardless the human variability. Moreover the use of an averaging indicator sensibly reduces the data fluctuation when compared to Fig.4. In the worst case the uncertainty is the $3 \%$ of the mean value, while in the early grade of the restenosis (ISR <50\%) it drops to less than $1.5 \%$.

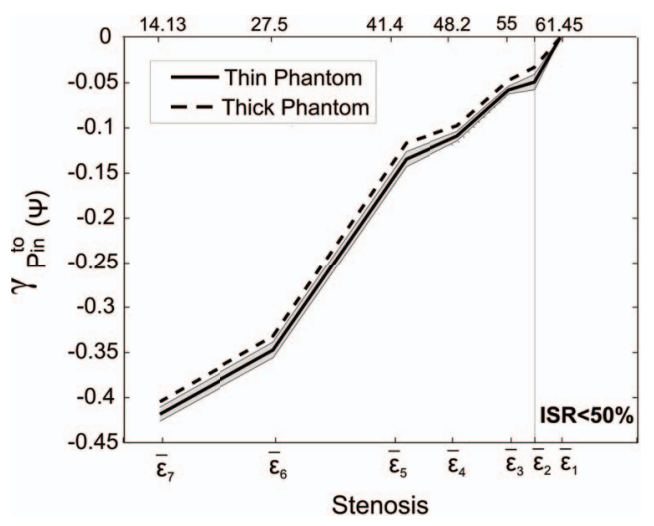

Figure 5. Normalized scale factor $\gamma$ of the turn on power for the two phantoms, on varying the liquid composition inside the carotid-like pipe.

Finally, by a linear fitting of the curves in Fig. 4 and 5, it is possible to estimate the percentage variation of all the previous power indicators referred to the healthy state $\bar{\epsilon}_{1}$ (Tab.II). In particular, a $11 \%$ of variation of the STENTag response may be achieved in the early restenosis $\left(\bar{\epsilon}_{1} \rightarrow \bar{\epsilon}_{2}\right)$ by monitoring
Table II

MEASURED RELATIVE CHANGES IN THE STENTAG RESPONSES

\begin{tabular}{|c|c|c|}
\hline & $\Delta P_{t o}^{\text {in }}(\%)$ & $\Delta \gamma_{P_{t o}^{\text {in }}}(\%)$ \\
\hline \hline $\bar{\epsilon}_{1} \rightarrow \bar{\epsilon}_{2}$ & $11 \%$ & $6 \%$ \\
\hline $\bar{\epsilon}_{1} \rightarrow \bar{\epsilon}_{7}$ & $56 \%$ & $42 \%$ \\
\hline
\end{tabular}

the changes of the turn-on power and more than $50 \%$ changes at the atherosclerotic restenosis $\left(\bar{\epsilon}_{1} \rightarrow \bar{\epsilon}_{7}\right)$.

\section{CONCLUSIONS}

In vitro experimentation seems to further corroborate the possibility to sense some inner biological processes by means of implanted passive RFID tags. These phenomena in evolution produce specific and macroscopic effects on the electromagnetic response of the radio-sensor, which are detectable by the available low-cost technology and with limited power budgets (maximum radiated power less than $1 W$ EIRP), making feasible to embed the required reader capability inside an hand-held device and even into a smart-phone.

In addition to the turn-on power, the RFID interrogation provides another independent measurement, the backscattered power which, if observed over the frequency, may provide really robust and effective metrics, rather immune to the specificity of patients and to the measurement modality.

Beside the promising results, this class of implanted RFID sensor tags is however still prone to considerable improvements concerning the interrogation modalities and instruments. Actual commercial UHF readers are designed to item labeling and not to sensing, and therefore significantly better performances are expected with dedicated receivers having a finer resolution in the power tuning and in the analog to digital converter.

\section{ACKNOWLEDGMENT}

Work developed under project PRIN-2008: MULTI-TAG funded by Italian Ministry of University. The authors wish to thank prof. Lorenzo De Medici of the Radiology Department of San Camillo Hospital, Roma, for inspiration and discussions.

\section{REFERENCES}

[1] Occhiuzzi, C.; Contri, G.; Marrocco, G.; , "RFID STENTag for passive vascular monitoring," Antennas and Propagation (EUCAP), Proceedings of the 5th European Conference on , vol., no., pp.3476-3478, 11-15 April 2011

[2] Brajesh K. Lal, Elias A. Kaperonis, Salvador Cuadra, Indravadan Kapadia, Robert W. Hobson II, "Patterns of in-stent restenosis after carotid artery stenting: Classification and implications for long-term outcome", Journal of Vascular Surgery, Volume 46, Issue 5, November 2007, Pages 833-840

[3] C. Gabriel, S. Gabriel, "Compilation of the Dielectric Properties of Body Tissues at RF and Microwave Frequencies", Internet document; URL: http://niremf.ifac.cnr.it/docs/DIELECTRIC/home.html

[4] G. Hartsgrove, A. Kraszewsky, A. Surowiec, "Simulated biological materials for electromagnetic radiation absorption studies", Bioelectromagnetics, vol. 8, n. 4, pp. 29-36, 1987 